\title{
Avaliação de Desempenho de um Controle Remoto: Teste de Usabilidade com Jovens Universitários
}

\section{Performace Evaluation Of a Remote Control: Usability Testing with University Students}

Mayara Ramos ${ }^{1}$, Isis Bastos Teixeira², Tamine Saccomori Lopes Dal Mago ${ }^{3}$, Alexandre Amorim dos Reis 4 


\section{Resumo}

O presente trabalho apresenta o teste de usabilidade de um controle remoto com usuários adultos jovens. $\mathrm{O}$ objetivo desse trabalho é observar a interação dos usuários com o objeto de estudo com foco no desempenho do individuo ao realizar a tarefa. As métricas de desempenho analisadas foram tempo de realização da tarefa, sucesso da tarefa, número de erros e número de reposicionamentos necessários para realizar a tarefa. Os resultados foram comparados entre homens e mulheres para avaliar se existe diferença no desempenho entre gêneros. A análise estatística dos dados demonstrou que homens e mulheres jovens não apresentam diferenças estatísticas significativas.

Palavras-Chave: Fatores humanos, usabilidade, avaliação de desempenho.

\section{Abstract}

The present paper presents the usability test of a remote control with young adults. The objective of this paper is to observe the user interaction with the object of study, focusing in the subject performance on task execution. The performance metrics analyzed were time on task, task success, number of errors, and number of positioning needed to perform task. The results were compared between men and women to evaluate if there is a performance difference among gender. Statistical analysis of the data showed that young men and women do not show significant differences.

Keywords: Human factors, usability, performance evaluation.

ISSN: 1808-3129

\footnotetext{
${ }^{1}$ Mestre pela Universidade do Estado de Santa Catarina - UDESC, Brasil,mayara.ramos@gmail.com

2 Universidade do Estado de Santa Catarina - UDESC, Brasil isis.bt@hotmail.com
}

${ }^{3}$ Graduada em Ciências Biológicas pela Universidade Federal de Mato Grosso do Sul, Brasil, taminedm@hotmail.com

4 Orientador, Prof. Dr. da Universidade do Estado de Santa Catarina - UDESC, Florianópolis - SC Brasil, alexandre.reis@pq.cnpq.br 


\section{INTRODUÇÃO}

Fatores humanos podem ser definidos como o desenvolvimento e aplicação do conhecimento sobre comportamento e a fisiologia humana no ambiente operacional. Aqueles que atuam na área realisam pesquisas e analise do desempenho humano, incluindo seus limites e habilidades. Os fatores humanos extraem conhecimentos de áreas e métodos diversos para desenvolver sistemas que são centrados no usuário (NEMETH, 2004).

Segundo Pheasant (2003), a abordagem ergonômica (ou dos fatores humanos) do design, pode ser resumida pelo principio do design centrado no usuário - se um objeto, um sistema ou um ambiente é projetado para o uso humano, então o seu design deve ser baseado nas características físicas e mentais dos seus usuários. Objetiva-se conseguir a melhor correlação entre o produto e seus usuários, no contexto da tarefa de trabalho a ser realisada.

O design centrado no usuário se relaciona diretamente com a usabilidade. Krug (2008) coloca que a usabilidade é garantir que algo funciona bem, que uma pessoa com habilidade e experiência comuns possa usar algo - seja um site da web, um caça a jato ou uma porta giratória - para seu propósito desejado sem causar frustração.

A usabilidade serve como um indicador de quão intuitivo é utilizar um produto e é um dos atributos de qualidade perceptível aos usuários. Essa característica é determinante no sucesso de um produto, ela influencia diretamente o interesse do usuário em fazer uso do produto (SILVA FILHO, 2010).

Nesse artigo será apresentado um teste de usabilidade exploratório com um objeto utilizado no cotidiano de grande parte da população - controle remoto. 0 objetivo desse teste é observar a interação dos usuários com o objeto, focando no desempenho do individuo ao realizar uma tarefa com o objeto de estudo. As métricas avaliadas nesse teste de usabilidade foram tempo de realização da tarefa, número de erros, sucesso da tarefa, e número de reposicionamentos necessários para realizar a tarefa. Com o resultados dessas medidas foram feitos testes estatísticos para comparar o desempenho entre homens e mulheres.

\section{USABILIDADE}

A NBR/ISO 9241-11 (ABNT, 2002) define usabilidade como: "Medida na qual um produto pode ser usado por usuários específicos para alcançar objetivos específicos com eficácia, eficiência e satisfação em um contexto específico de uso". Sendo que, para a norma:

- eficácia: precisão e completude com as quais usuários alcançam objetivos específicos;

- eficiência: recursos gastos em relação à precisão e abrangência com as quais usuários atingem objetivos;

- satisfação: ausência do desconforto e presença de atitudes positivas para com o uso de um produto;

- contexto de uso: usuários, tarefas, equipamento (hardware, software e mate- 
riais), e o ambiente físico e social no qual um produto é usado.

Jordan (1998) destaca um ponto importante da definição de usabilidade que deixa claro que não se trata simplesmente de uma propriedade do produto isolado, mas que dependerá também de quem está usando o produto, o objetivo que eles estão procurando alcançar e o ambiente em que o produto está sendo usado. $O$ autor conclui que a usabilidade é a propriedade de interação entre um produto, um usuário e a tarefa, ou conjunto de tarefas, que o usuário está tentando completar.

\subsection{Testes de usabilidade}

Segundo Krug (2008), o intuito dos testes de usabilidade é bastante simples: se quiser saber se um software, site da web ou controle remoto de um aparelho é, suficientemente, fácil de usar, assista algumas pessoas enquanto tentam usá-lo e observe onde elas têm dificuldade. A seguir, conserte-o e teste novamente.

Nessa mesma linha, Nemeth (2004) coloca que a avaliação da usabilidade segue um padrão de três passos. Apresentar a usuários reais um protótipo ou o produto real. Convidá-los a realizar uma tarefa com o produto. Observar o que o usuário fala (ou não fala) e faz (ou não faz).

A avaliação de usabilidade pode ser realizada para determinar se há dificuldades com o uso de um produto ou para descobrir oportunidades para melhoria. Nesse processo, aqueles que estão fazendo as observações, com frequência descobrem novas maneiras de como o usuário se comporta enquanto executa uma tarefa (NEMETH, 2004).

Durante um teste de usabilidade, observadores assistem os participantes realizarem uma determinada tarefa com um produto em um ambiente de teste. Isso é o que difere os testes de usabilidade de outros métodos de design centrado no usuário. Essa visão de testes de usabilidade permite uma grande variedade em técnicas de testes, podendo ser bastante informal ou formal (LEWIS, 2006).

A realização de um teste de usabilidade, segundo Dumas e Loring (2008), envolve um participante, um moderador, e deve apresentar as seguintes tarefas: saudar o participante, explicar-lhe seus direitos e solicitar a assinatura de um termo de consentimento, explicar o procedimento de teste, guiar o participante por uma série de atividades selecionadas usando o produto (geralmente enquanto pensa em voz alta - thinking aloud), gravar os dados de uma ou mais formas, e solicitar ao participante que resuma sua experiência.

\subsection{Métrica de usabilidade}

A métrica é uma maneira de mensurar ou avaliar um fenômeno ou objeto. A usabilidade tem um conjunto de medidas específicas para ela: sucesso da tarefa, satisfação do usuário e erros, entre outros. Todas as medidas de usabilidade devem ser observáveis de alguma forma, seja direta ou indiretamente. Todas essas medidas precisam ser quantificáveis - elas têm que ser transformadas em um número ou contadas de alguma forma. Todas as medidas de usabilidade também precisam que o que está sendo medido represente um aspecto da experiência do usuário, apresentada 
em um formato numérico (TULLIS; ALBERT, 2008).

A métrica de usabilidade revela algo sobre a interação entre o usuário e o objeto: algum aspecto da eficácia, eficiência ou satisfação. A mensuração de usabilidade mede algo sobre pessoas e seu comportamento ou atitudes; como as pessoas são muito diversas e adaptáveis, algumas vezes se encontram desafios na métrica de usabilidade. Algumas coisas não são consideradas medidas de usabilidade, como preferências e atitudes não vinculadas a experiência de usar algo (TULLIS; ALBERT, 2008).

Métricas de desempenho são a melhor forma de avaliar eficácia e eficiência de diferentes produtos. Se os usuários estão cometendo muitos erros, se sabe que tem oportunidades de melhoria. Caso os usuários estejam muito mais tempo que o planejado para executar uma tarefa, a eficiência pode ser muito aprimorada. As métricas de desempenho são a melhor forma de saber quão bem os usuários realmente estão usando um produto (TULLIS; ALBERT, 2008).

Tullis e Albert (2008) apresentam cinco métricas básicas para o desempenho: sucesso da tarefa, tempo de realização da tarefa, erros, eficiência e facilidade de aprendizagem. Jordan (1998) apresenta as métricas de usabilidade divididas entre as medidas e eficácia, eficiência e satisfação; as medidas de eficácia são: conclusão da tarefa, qualidade de resultado; as de eficiência são: desvios de caminho crítico, taxa de erros, tempo de realização da tarefa, carga de trabalho mental.

\section{MATERIAIS E MÉTODOS}

Essa pesquisa se trata de uma pesquisa exploratória, focando na interação dos usuários com o objeto de estudo, e coletar medidas de desempenho na realização de uma atividade. A atividade realizada pelos sujeitos pesquisados foi pressionar teclas determinadas de um controle remoto segurando o objeto de forma confortável ao usuário. Nesse teste foram medidos sucesso da tarefa, número de erros, tempo de realização da tarefa (medidas de eficácia), e número de reposicionamentos necessários para efetuar a tarefa (medida de eficiência).

\subsection{Indivíduos do estudo}

Os indivíduos desse estudo foram homens e mulheres de 18 a 40 anos pareados por sexo. Os indivíduos são residentes da Grande Florianópolis. Os participantes dessa pesquisa não deveriam apresentar registros de doenças musculoesqueléticas, ou baixa acuidade. Os indivíduos do estudo participaram do experimento como parte de uma atividade realizada na disciplina de Ergonomia aplicada ao Design Industrial ministrada no curso de graduação em Design Industrial da Universidade do Estado de Santa Catarina (UDESC).

Por se tratar de um estudo que envolve seres humanos essa pesquisa foi submetida à avaliação do Comitê de Ética da UDESC, estando em conformidade com a Resolução 466/2012/CNS/MS o mesmo foi aprovado. Os indivíduos que participaram do estudo foram apresentados e preencheram ao Termo de Consentimento Livre Esclarecido (TCLE) e o Consentimento para fotografias, vídeos e gravações. Todos os testes e coletas de dados foram realizados em um dia pré-agendado com os sujeitos 
que aceitaram participar da pesquisa.

\subsection{Instrumentos do estudo}

Para o estudo foi utilizada uma ficha cadastral para coletar dados como nome, idade, se possui doenças musculoesqueléticas, qual a mão dominante e questionário pós-teste (cinco questões de respostas binomiais), controle remoto de grande presença no mercado (controle de operadora de TV a cabo - NET, Figura 4.1), filmadora de alta resolução e tripé.

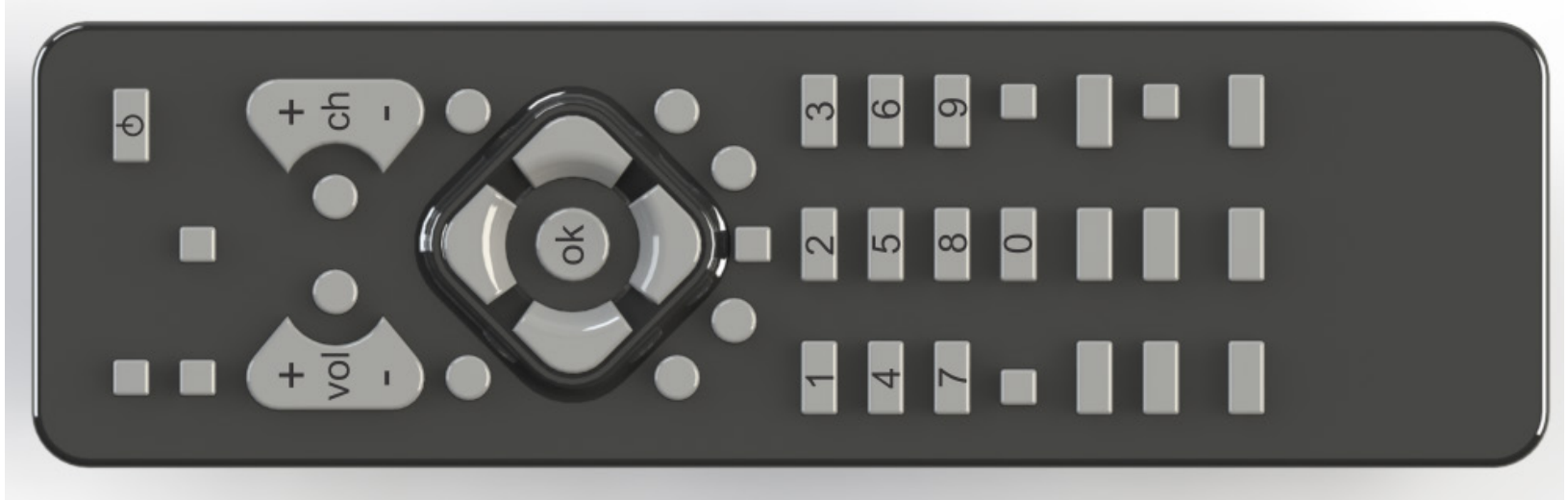

Figura 3.1: Representação do controle remoto utilizado. Fonte: Elaborado pelos autores.

\subsection{Procedimentos de coleta de dados}

A coleta de dados foi realizada no dia 2 de outubro de 2013, no laboratório de pesquisas ergonômicas, do Centro de Artes. Para essa etapa foi solicitado aos jovens para que realizassem a tarefa de manuseio de controle remoto. A tarefa solicitada aos usuários foi o acionamento de uma série de botões em localizações variadas, fazendo com que o usuário passe por todas as faixas do controle.

O teste de usabilidade seguiu a seguinte sequencia:

1. Apresentação e assinatura do TCLE e consentimento de uso de imagens e dados cadastrais.

2. Posicionamento do sujeito na posição sentada, ficando de lado para a câmera filmadora.

3. Realização da tarefa com a sequência de acionamentos dos botões: ligar, 2 , 5, 0, ok, ch+, vol-.

4. Aplicação de questionário de pós-teste da percepção do usuário sobre a atividade.

\subsubsection{Análise dos dados}

Esse teste foi filmado para análise da atividade, onde foram observadas as variáveis medidas nesse estudo (sucesso da tarefa, número de erros, tempo de realização da tarefa, e número de reposicionamentos necessários para efetuar a tarefa). 
Para a métrica do sucesso da tarefa, se tem como foco os movimentos realizados na manipulação do objeto, sendo assim, o sucesso da tarefa se dará pelo pressionamento apenas das teclas indicadas. Se o indivíduo ao realizar a tarefa cometendo erros, como acionar um botão que não corresponde ao solicitado, a tarefa será de realização parcial com erros. O indivíduo que não conseguir realizar a tarefa pressionando apenas o botão correspondente ao solicitado, acionando mais de um botão; ou no caso dos botões de canal e volume em que o mesmo botão é dividido para aumentar ou diminuir, se for pressionado no meio do botão (região neutra) a tarefa não foi realizada com sucesso. É considerado erro quando o indivíduo pressiona um botão que não corresponde ao solicitado. Para a métrica tempo de realização da tarefa o tempo é contado a partir da solicitação do acionamento do botão ligar. O número de reposicionamentos necessários se dá pelo número de vezes que o indivíduo muda o local de apoio do controle remoto na mão.

\subsubsection{Tratamento estatístico dos dados}

Para tratamento dos dados foi primeiramente apresentado uma caracterização dos sujeitos do estudo através da estatística descritiva. Posteriormente foram comparados os resultados de homens e mulheres das métricas: tempo de realização da tarefa, número de erros, sucesso da tarefa e número de reposicionamentos necessários para efetuar a tarefa. A comparação de resultados foi feito por meio de testes estatísticos de hipótese.

Para a métrica de tempo de realização da tarefa, foi realizado o teste $t$ independente, caso os resultados apresentem distribuição normal e simétrica. Para as demais medidas foi utilizado o teste não paramétrico teste $U$ de Mann Whitney. Buscou-se um nível de confiabilidade dos dados de $95 \%$, sendo nível de significância empregado de $5 \%(\operatorname{sig}=0,05)$.

\section{RESULTADOS E DISCUSSÕES}

Esse estudo foi realizado com 12 estudantes, sendo 6 homens e 6 mulheres, desses 2 eram canhotos ( 1 homem e 1 mulher), apresentando uma média de idade de 22,6 anos. Durante o teste 4 dos sujeitos cometeram erros. Dos participantes que cometeram erros, 3 deles tiveram um erro (trocaram o botão de ch+ pelo botão de seta para cima) e um único participante teve dois erros (trocou o botão ch+ pela seta para cima e o botão vol- pela seta para esquerda).

As tabelas a seguir apresentam os resultados descritivos das métricas sucesso da tarefa (Tabela 4.1), tempo da tarefa (Tabela 4.2), número de reposicionamentos do controle necessários para realizar a tarefa (Tabela 4.3), e das respostas das questões pós-teste (Tabela 4.4). 
Tabela 4.1: Sucesso da Tarefa

\begin{tabular}{|c|c|c|c|}
\hline \multirow{2}{*}{ Realizou a Tarefa } & \multicolumn{2}{|c|}{ Sexo } & \multirow{2}{*}{ Total } \\
\cline { 2 - 3 } & Feminino & Masculino & \\
\hline Sucesso & 4 & 4 & 8 \\
\hline Parcial - com erros & 2 & 2 & 4 \\
\hline Total & 6 & 6 & 12 \\
\hline
\end{tabular}

Fonte: Elaborado pelos autores, com base na pesquisa realizada.

Tabela 4.2 Tempo de realização da tarefa.

\begin{tabular}{|l|c|c|c|c|c|c|c|}
\hline $\begin{array}{l}\text { Tempo } \\
\text { tarefa(s) }\end{array}$ & T. Mín. & T. Máx. & Média & Mediana & DP & IClower & IC upper \\
\hline Homens & 8 & 11.33 & 11 & 3.7 & 7.43 & 15.24 & \\
\hline Mulheres & 8 & 17 & 13 & 13.5 & 13.5 & 9.68 & 16.32 \\
\hline Todos & 8 & 12.16 & 12 & 3.4 & 3.4 & 14.33 & \\
\hline
\end{tabular}

Fonte: Elaborado pelos autores, com base na pesquisa realizada.

Tabela 4.3: Número de reposicionamentos para realizar a tarefa

\begin{tabular}{|l|c|c|c|c|c|c|c|}
\hline $\begin{array}{l}\text { No reposicio- } \\
\text { namentos }\end{array}$ & Mín. & Máx. & Média & Mediana & DP & IC lower & IC upper \\
\hline Homens & 0 & 1 & 0.67 & 1 & 0.51 & 0.12 & 1.21 \\
\hline Mulheres & 0 & 3 & 1 & 1 & 1.09 & -0.15 & 2.15 \\
\hline Todos & 0 & 3 & 0.83 & 1 & 0.83 & 0.3 & 1.36 \\
\hline
\end{tabular}

Fonte: Elaborado pelos autores, com base na pesquisa realizada.

Tabela 4.4: Frequências respostas questionário pós-teste

\begin{tabular}{|l|c|c|c|c|c|c|}
\hline \multicolumn{1}{|c|}{ Questão } & \multicolumn{2}{c|}{ Homens } & \multicolumn{2}{c|}{ Mulheres } & \multicolumn{2}{c|}{ Total } \\
\cline { 2 - 8 } & Sim & Não & Sim & Não & Sim & Não \\
\hline Conhecia o controle remoto? & 5 & 1 & 6 & 0 & 11 & 1 \\
\hline Sentiu neces. de reposicionar o controle? & 3 & 3 & 5 & 1 & 8 & 4 \\
\hline Teve dificuldade de ident. alguma das teclas? & 0 & 6 & 0 & 6 & 0 & 12 \\
\hline Teve desconforto para efetuar a tarefa? & 1 & 5 & 3 & 3 & 4 & 8 \\
\hline Teve outra dificuldade na realização da tarefa? & 0 & 6 & 1 & 5 & 1 & 11 \\
\hline
\end{tabular}

Fonte: Elaborado pelos autores, com base na pesquisa realizada.

Esses dados apontam que, para esses indivíduos, o sucesso da tarefa foi o mesmo para homens e mulheres com o mesmo número de sucessos totais e parciais com erros. Para o tempo de tarefa, pela média, os homens aparentam a realizar a tarefa em menos tempo, sendo que o menor tempo encontrado (8s) é o mesmo para homens e mulheres e o valor máximo apresenta uma diferença de apenas 1 segundo. $O$ número de reposicionamentos indica, pelos números máximos e mínimos encontrados, que as mulheres tiveram um número maior de reposicionamentos.

A Tabela 4.5 mostra o resultado do teste $t$ independente para a variável tempo. Como o resultado para sig (significância/probabilidade) foi maior que 0,05 adota-se a hipótese nula, a média do tempo de realização da tarefa entre homens e mulheres jovens não apresentam diferenças significativas. 
Tabela 4.5: Resultado teste $\mathrm{t}$ independente para variável tempo de realização de tarefa.

\begin{tabular}{|c|c|c|c|c|c|c|}
\hline Sig & $T$ & $\begin{array}{c}\text { Mean Diffeer- } \\
\text { ence }\end{array}$ & DP & $\begin{array}{c}\text { Std. Error } \\
\text { Difference }\end{array}$ & \multicolumn{2}{|c|}{$95 \%$ IC of the Dif } \\
\hline 0.423 & 0.836 & 1.667 & 10 & 1.994 & Lower & Upper \\
\cline { 5 - 8 } & & & & & -2.777 & 6.111 \\
\hline
\end{tabular}

Fonte: Elaborado pelos autores, com base na pesquisa realizada.

A Tabela 4.6 apresenta os resultados dos testes das variáveis não paramétricas: sucesso da tarefa, número de erros e número de reposicionamentos para realização da tarefa.

Tabela 4.6: Resultado teste U de Mann-Whitney

\begin{tabular}{|l|l|l|l|}
\hline & Realizou a tarefa & Número de erros & $N^{\circ}$ de reposocionamentos \\
\hline Mann-Whitney U & 18.000 & 17.000 & 16.000 \\
\hline Sig. & 1.000 & 0,937 & 0.818 \\
\hline
\end{tabular}

Fonte: Elaborado pelos autores, com base na pesquisa realizada.

O resultado do teste de hipótese para as variáveis do sucesso da tarefa, número de erros e número de reposicionamentos para realizar a tarefa, também indicam a adoção da hipótese nula, indicando que homens e mulheres não apresentam resultados com diferença significativa para essas métricas.

\section{CONCLUSÃO}

Objetos como o controle remoto estão presentes diariamente nas atividades da população. Objetos como este, por serem simples e de bastante familiaridade com usuários, podem continuar sendo lançados no mercado seguindo o padrão atual. Testes de usabilidade podem demonstrar que esses objetos ainda necessitam melhorias.

O teste apresentado nesse estudo buscou fazer uma analise exploratória do desempenho de jovens adultos, procurando obter dados mensuráveis da interação do usuário com o objeto de estudo. Em geral a comparação do desempenho de homens e mulheres não apresentaram diferença significativa em nenhuma das métricas.

A métrica de sucesso da tarefa os resultados da descritiva e do teste $U$ de Mann-Whitney foram similares, já que os resultados dos homens e mulheres que participaram do teste foram o mesmo. A alta taxa de sucesso da tarefa pode ser relacionada à maioria dos usuários conhecerem previamente o controle remoto (apenas um sujeito não tinha familiaridade).

A métrica tempo da tarefa nos dados descritivos apresentou algumas diferenças, mas não foram significativas no teste de hipótese (teste $t$ independente). A diferença apresentada no número de reposicionamentos necessários para efetuar a tarefa nos sujeitos pesquisados (estatística descritiva) pode ser devido à diferença antropométrica do tamanho da mão dos homens que tende a ser maior do que das mulheres, e assim a área de alcance do dedo sendo utilizado para efetuar os acionamentos tam- 
bém é maior.

Os erros registrados em quatro dos sujeitos pesquisados foram erros em que o sujeito não pressionou o botão solicitado apartando outro botão. As respostas do questionário pós-teste demonstram que os usuários não apresentaram dificuldades para identificar os botões, no entanto quatro desses participantes cometeram erros por não identificarem corretamente os botões solicitados. Esses erros podem ter ocorrido pela similaridade cognitiva das teclas e pelo posicionamento dos botões com as setas, que em alguns controles com botões similares em posicionamento similar são botões com as funções de volume e canal.

As questões relacionadas ao conforto e dificuldades de realizar a tarefa indica que a maior parte dos participantes não sentiram desconforto ou dificuldade para realizar a tarefa. Dos que responderam sentir desconforto ou dificuldade estava relacionado à necessidade de mudar o posicionamento do controle remoto para completar a tarefa.

Os resultados encontrados com o público de adultos jovens pode não ser o mesmo com outras faixas etárias da população. Em trabalhos futuros serão feitas análises comparativas do desempenho no uso de controles remotos entre jovens e idosos (faixa da população que tem algumas limitações de movimento e tempo de reação devido ao envelhecimento). Também se pretende comparar os resultados de testes tradicionais de usabilidade com o resultado de testes de analisew ergonômica virtual. 


\section{REFERÊNCIAS}

Associação Brasileira de Normas Técnicas (ABNT). NBR 9241-11: Requisitos ergonômicos para trabalhos de escritórios com computadores, Parte 11 - Orientações sobre usabilidade. Rio de Janeiro; 2002.

DUMAS, J. S.; LORING, B. A. Moderating usability tests: principles and practice for interacting. Amsterdam: Elsevier, 2008.

JORDAN, Patrick W. An introduction to usability. London: Taylor \& Francis, 1998.

KRUG, Steve. Não me faça pensar: uma abordagem de bom senso à usabilidade na web. 2. ed. Rio de Janeiro: Alta Books, 2008.

LEWIS, J. R. Usability Testing. In: SALVENDY, Gavriel. Handbook of Human Factors and Ergonomics. Hoboken, NJ: John Wiley \& Sons, cap. 49, p. 1275-1316, 2006.

NEMETH, C. P. Human Factors Methods for Design: Making Systems Human-Centered. Boca Raton, FL: CRC Press, 2004.

NET. - O mundo é dos nets. www.netcombo.com.br. Acessado em: 27/03/2014.

PHEASANT, S. Bodyspace: Anthropometry, Ergonomics and the Design of Work. 2a ed. Londres: Taylor \& Francis, 2003.

SILVA FILHO, A. M. da. Avaliação de Usabilidade: "Separando o joio do trigo". Revista Espaço Acadêmico, No 112, set. 2010.

TULLIS, Tom; ALBERT, Bill. Measuring the user experience: collecting, analyzing, and presenting usability metrics. Boston, MA: Morgan Kaufmann, 2008. 